\title{
Estudos urbanos e geometria fractal
}

\author{
Niara Palma \\ Universidade de Santa Cruz do Sul, Rua Jerônimo Coelho 267, CEP 90010241 Bairro \\ Centro Histórico, Porto Alegre, Rio Grande do Sul, Brasil. \\ Email: niara.palma.br@gmail.com
}

Artigo revisto recebido a 24 de Janeiro de 2014

\begin{abstract}
Resumo. Muitas metodologias convencionais utilizadas no estudo da forma urbana não têm considerado o grau de irregularidade dos perímetros urbanos nem toda a complexidade morfológica, pois tratam a forma das cidades dentro do âmbito da geometria euclidiana. Este artigo explora a aplicação da geometria fractal ao estudo de áreas urbanas, centrando-se num conjunto de aspetos da forma urbana relacionados com a fragmentação dos tecidos urbanos, a presença de vazios urbanos e, por fim, a 'auto-semelhança' em diferentes escalas de observação. Esta análise desenvolve-se a partir do trabalho de investigação desenvolvido por Frankhauser desde os anos 90. A análise fractal é aplicada a um conjunto de cinco cidades no Vale do Rio Pardo, no Rio Grande do Sul, Brasil. Ao longo dos últimos anos, este conjunto de cidades tem crescido de forma desordenada, sendo que o desenvolvimento acelerado e a ampliação do perímetro urbano originaram uma ocupação rarefeita com grandes impactos sobre os tecidos urbanos.
\end{abstract}

Palavras-chave: estudos urbanos, geometria fractal, dimensão fractal, autosemelhança, Vale do Rio Pardo

A evolução da estrutura urbana pode ser representada como um sistema que progride em passos discretos. Cada estado alcançado depende das relações funcionais, da história e do processo de evolução anterior. Neste artigo considera-se a cidade como um sistema dinâmico, em constante transformação, onde a estrutura urbana é formada ao longo do tempo, gerando potenciais de atratividade capazes de catalisar a transformação espacial. A estrutura urbana é, então, o resultado de uma série de relações espaciais e socioeconómicas que se complementam e que se manifestam fisicamente no espaço construído. Assim como as decisões de localização de atividades (decisões que deixam marcas importantes na composição do espaço urbano), também as inovações de uso interferem no crescimento e na densidade de ocupação, estruturando a organização e a complexidade do sistema urbano.

Os estudos urbanos consideram $\mathrm{o}$ crescimento da cidade, as suas transformações, os padrões que nela vão emergindo - as novas ordens e estruturas. Neste sentido, os investigadores nesta área científica têm trabalhado no desenvolvimento de modelos que possam refletir, de uma forma cada vez mais refinada, as características dinâmicas do sistema urbano. $\mathrm{O}$ modelo, sendo uma representação simplificada da realidade, permite a 'descrição' de um modo sistémico. Este processo ocorre no sentido de extrair do sistema urbano concreto os aspetos fundamentais de cada fenómeno estudado e de inferir sobre as principais relações existentes.

A análise do crescimento urbano pode requerer formas quantitativas de 'descrição' que permitam a identificação de diferentes padrões de ocupação. Para uma reflexão sobre as diferentes formas de crescimento urbano deverá ser considerada a forma destes padrões, a sua representação e a possibilidade de comparação da sua forma, podendo relacionar diferentes sistemas urbanos ou 
manter o foco na análise da evolução de apenas um sistema ao longo do tempo.

Em muitos casos, as áreas urbanas são formadas por clusters, de diferentes tamanhos, distribuídos espacialmente de forma não homogénea ao longo de vales, linhas de transporte ou áreas menos acessíveis ocupadas de maneira esparsa. Estas situações levam os investigadores e os profissionais a procurar novos modos de 'descrição' da forma urbana, abrindo-se aqui um espaço para a utilização da geometria fractal.

\section{Geometria fractal}

Ao longo dos anos 70 e 80, Mandelbrot desenvolveu a geometria fractal, uma descrição sistemática e abrangente da linguagem matemática subjacente aos processos e formas naturais - ver por exemplo, o livro seminal The fractal geometry of nature (Mandelbrot, 1982). Em 1975, Mandelbrot introduziu o termo fractal, do latim fractus, que significa 'fragmentado'. A própria palavra 'fractal' contém um importante aspeto visual dessas estruturas matemáticas, e de muitas estruturas naturais - a sua aparência irregular e fragmentada (Joye, 2011).

Uma característica importante das formas fractais é que este aspeto fragmentado surge em diferentes escalas da estrutura - uma propriedade designada como 'autosemelhança'. Talvez a característica mais importante de um fractal seja a 'dimensão fractal'. Enquanto na geometria Euclidiana a dimensão fornece uma informação sobre o espaço ocupado pelo objeto, na geometria fractal a dimensão (fractal) representa também o nível de irregularidade de um objeto (Mandelbrot, 1991). Quanto maior for a irregularidade de uma forma, maior será a sua dimensão fractal. Essa característica da dimensão fractal torna-a numa ferramenta muito útil para a apreensão das características individuais de uma forma. Torna-a, por exemplo, uma ferramenta útil para perceber a expansão de novas áreas urbanas a partir de uma área inicial.

$\mathrm{Na}$ geometria Euclidiana, um ponto tem dimensão 0 , uma linha tem dimensão 1 , um quadrado ou um retângulo têm dimensão 2 e um volume no espaço é 3-dimensional. Pelo contrário, um fractal num plano terá uma dimensão entre 1 e 2, e um fractal no espaço terá uma dimensão entre 2 e 3 . Os fractais têm uma dimensão não-integral pois ocupam mais espaço do que uma simples linha - a primeira dimensão - mas não ocupam todo o plano - a segunda dimensão (Joye, 2011).

Falconer (1991) sustenta que para um objeto ser considerado um fractal deverá cumprir um conjunto de requisitos: ser autosimilar (um intervalo entre 0 e $1 / 2$ deve ser igual a um intervalo entre $1 / 2$ e 1 ); ser demasiado irregular para poder ser descrito pela geometria tradicional; possuir dimensão fractal, que será normalmente superior à dimensão Euclidiana; poder ser obtido através de um procedimento recursivo; e, por fim, não variar com a escala.

A geometria fractal tem vindo a ser utilizada em diversas áreas do conhecimento como o estudo de sistemas caóticos (por exemplo, o padrão de formação de nuvens), a caracterização de objetos, a análise e o reconhecimento de padrões em imagens, a análise de texturas e a medição do comprimento de curvas.

Desde os anos 80 a geometria fractal tem sido utilizada para a análise de fenómenos urbanos, destacando-se: na década de 90, os trabalhos de Allen (1997), Batty e Longley (1994) e Frankhauser (1994, 1998); na primeira década do século $\mathrm{XX}$, os textos de Batty (2003), Benguigui et al. (2008), Cooper e Oskrochi (2008), Frankhauser e Pumain (2000), Frankhauser (2002) e Weidlich (2000); e nos primeiros anos da presente década, Feng e Chen (2010) e Terzi e Kaya (2011). No Brasil esta abordagem foi já utilizada, na área da geografia, por Azevedo e Christofoletti (2007) e Trentin (2012).

Batty e Longley (1994) consideram que do mesmo modo que o crescimento natural, também o crescimento urbano envolve adição e subtração de unidades básicas, células ou partículas. No caso das cidades, estas unidades podem ser individuais, residências, firmas, ligações de transporte e outros. As cidades crescem através da acumulação destas unidades básicas. Estes padrões podem existir em escalas maiores e indicar auto-similaridade em escalas que emergem do processo de crescimento. 
Considera-se que dentro de cada processo de crescimento existem códigos que determinam o modo como a organização destas unidades básicas de desenvolvimento urbano define o seu crescimento e forma, repetindo-se em diferentes escalas. Deste modo a repetição de padrões torna-se um elemento natural no crescimento urbano.

\section{Modelos clássicos}

A propriedade mais básica de um objeto fractal é ele ser, por definição, multi-escalar. Esta característica resulta do procedimento utilizado para construir um fractal teórico, baseado na repetição da mesma operação, definida pelo objeto gerador, em escalas cada vez menores. Ao mesmo tempo, o limite deste objeto geométrico torna-se cada vez mais complexo pela inclusão de um número crescente de tentáculos (Frankhauser, 2004). A Figura 1 ilustra a geração do chamado 'Tapete de Sierpinski' (figura plana desenvolvida por Sierpinski).

Neste exemplo a estrutura geométrica permanece confinada á área do quadrado inicial. O mesmo tipo de objeto geométrico pode ser também construído pela adição de quadrados de uma determinada dimensão e de acordo com uma regra de geração, dando origem a um objeto cada vez mais complexo.

Segundo Frankhauser (2004), este exemplo pode ser, de algum modo, relacionado com um processo de expansão urbana onde, a partir de um núcleo inicial, são adicionadas novas áreas ao longo de quatro eixos de saída da cidade. Numa fase seguinte, a expansão continua ao longo dos eixos principais, mas também ao longo de eixos secundários dando origem a uma rede viária hierarquizada (Figura 2).

Por outro lado, a Figura 3 poderá sugerir áreas metropolitanas formadas por assentamentos de dimensão variada, desconectados, distribuídos de maneira desigual sobre o espaço. Novamente, a estrutura dos fractais torna-se mais e mais complexa a cada nova iteração.

Os fractais, regulares e irregulares, parecem assim oferecer uma base interessante para o estudo de assentamentos urbanos. Em seguida, serão considerados os espaços construídos da cidade como os

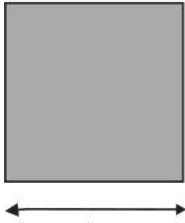

$\mathrm{L}$
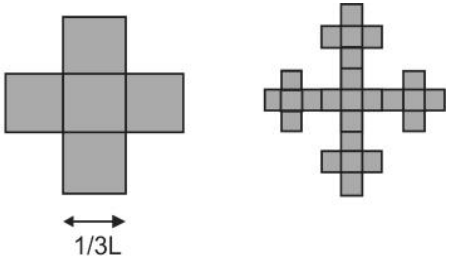

Figura 1. Geração de um Tapete de Sierpinski. Em cada passo, cada quadrado é substituído por $n=5$ quadrados com comprimento reduzido pelo fator $r=1 / 3$.

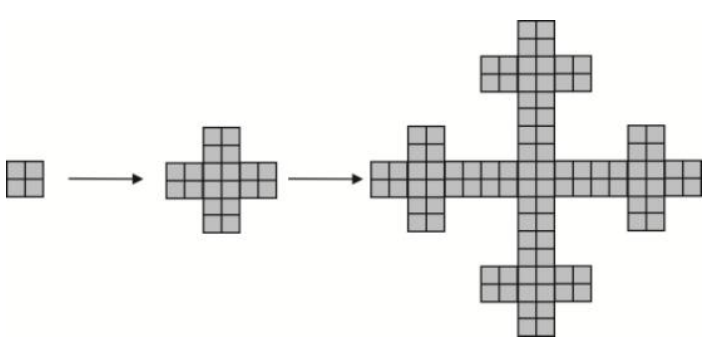

Figura 2. Geração de um fractal pela adição de elementos (relacionável com a rede viária).
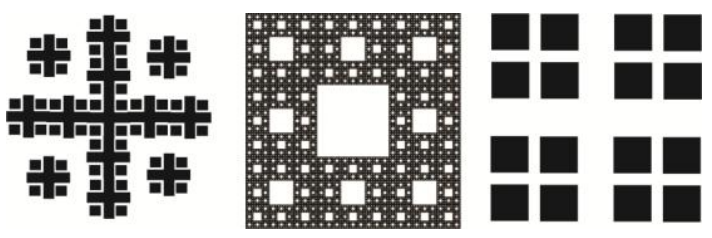

Figura 3. Um Tapete de Sierpinski consistente de uma série de clusters (a), um compacto com hierarquia de espaços abertos (b) e um tipo Poeira de Fournier que remete uma rede viária intra-urbana (c).

elementos constitutivos da 'massa' de uma estrutura fractal. Este processo envolve a contagem do número de pontos ocupados, que se encontram a uma determinada distância de cada ponto ocupado, calculandose em seguida a correlação entre eles. $\mathrm{O}$ processo inicia-se com apenas um ponto (um pixel), sendo de seguida ampliada a escala de análise até se cobrir a figura como um todo.

A dimensão mede o grau de concentração dos espaços ocupados através de diferentes escalas, ou mais precisamente, o decaimento relativo na massa em qualquer distância do ponto onde a massa é concentrada. Neste sentido, é possível perceber em detalhe a distribuição dos pontos ocupados na forma em análise. Como foi já referido, para as formas perfeitas, usualmente estudadas pela 
geometria Euclidiana, o valor estimado de dimensão fractal é muito próximo de 1 .

A dimensão fractal de uma superfície uniformemente ocupada será igual a 2 , pois essa não possui irregularidades. Note-se que no modelo de Frankhauser (2004), aqui utilizado para o cálculo de dimensão fractal, o valor é estimado segundo o número de pixels ocupado em cada figura, representada como uma forma dentro de um limite quadrado (geração de imagens que ainda deixam espaços em branco no seu limite). Devido a esta característica de leitura do modelo, os valores não são exatamente iguais a 2 .

Naturalmente, os assentamentos urbanos não são construídos como as figuras aqui apresentadas. Como tal, Frankhauser (2004) sugere diferentes métodos para verificar o quanto um padrão observado é, de facto, estruturado de acordo com a lógica fractal. Estes métodos reproduzem a lógica de interação medindo a distribuição dos espaços ocupados ou do comprimento da fronteira através de múltiplas escalas. Este tipo de análise foi utilizado para o estudo de diferentes tipos de ocupação, especialmente tipos considerados 'amorfos', com fronteiras 'suaves' ou 'dendríticas'. Quando a forma em análise não é uma figura geométrica perfeita, ou é preenchida de forma irregular, a sua dimensão fractal assume um valor compreendido entre 1 e 2. A Figura 4 compara uma forma circular perfeita (perímetro), com uma forma circular com preenchimento irregular e, ainda, com uma forma circular com a superfície uniformemente ocupada.

$\mathrm{Na}$ secção seguinte descreve-se uma aplicação a um conjunto de cidades do Vale do Rio Pardo. A aplicação centra-se no perímetro urbano e nos vazios internos, e tem em vista a identificação do tipo de ocupação presente em cada cidade. Foi utilizado o software Fractalyse desenvolvido no centro de investigação ThéMA num projeto coordenado por Frankhauser e Tannier. Será aplicada a medida de dimensão fractal, do tipo correlação, onde cada ponto é rodeado por uma janela de pequena dimensão.

Contabiliza-se o número de pontos ocupados dentro de cada janela, sendo que, em seguida, a mesma operação é aplicada para janelas cada vez maiores. Se houver

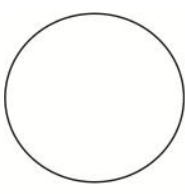

DF:0,9825

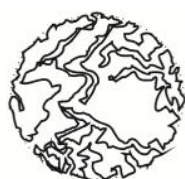

DF: 1,748

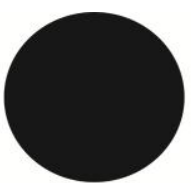

DF:1,899
Figura 4. Dimensão Fractal de um círculo segundo seu perímetro, preenchimento irregular e totalmente preenchido.

alteração de pontos proporcionalmente à escala da nova janela (correlação positiva), a forma é considerada uniforme. Por outro lado, se o número de pontos não for proporcional à mudança da escala de crescimento das janelas utilizadas, está-se em presença de uma forma fragmentada $\mathrm{e}$ irregular, como mostra a Figura 5. Em cada gráfico, o eixo $\mathrm{X}$ representa o lado da janela $\varepsilon=(2 i+1)$, o eixo Y representa a média de pontos contados por janela e o parâmetro principal é o tamanho do lado da janela $\varepsilon$.

\section{Caso de estudo: cinco cidades no Vale do Rio Pardo}

\section{Escala regional e urbana}

Esta secção apresenta a aplicação da geometria fractal a cinco cidades do Vale do Rio Pardo, no Rio Grande do Sul: Estrela, Lajeado, Santa Cruz do Sul, Venâncio Aires e, por fim, Vera Cruz. O Vale do Rio Pardo é uma região no Rio Grande do Sul - uma das 27 unidades federativas Brasileiras, localizada no extremo sul do país. Reside nesta região (com uma área de $13250 \mathrm{~km}^{2}$ ) uma população de perto de 450000 habitantes (dados da Fundação de Economia e Estatística do Rio Grande do Sul) distribuída por um vasto conjunto de cidades de pequena e média dimensão. A rede de cidades do Vale do Rio Pardo é apresentada na Figura 6, onde se identifica as que serão parte desta análise. Embora a figura destaque apenas as estradas principais, pode-se perceber a existência de uma rede formada por essas estradas e pelo conjunto de vias secundárias utilizadas diariamente pela população que transita entre estas cidades em função das relações de trabalho, estudo e transporte de mercadorias.

De entre as cinco cidades selecionadas 


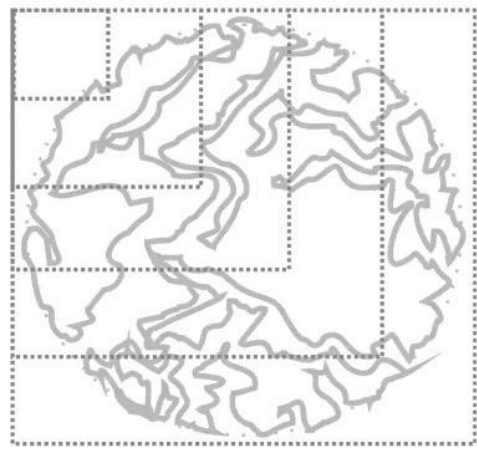

Dimensão Fractal: 1,748

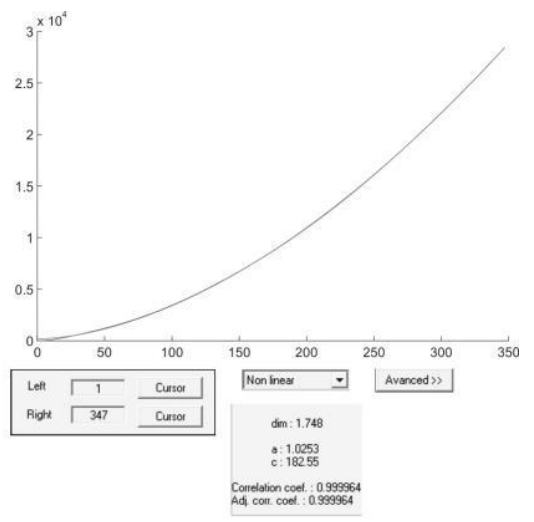

Figura 5. Ampliação da janela para a contagem de pontos existentes em cada escala considerada até preencher toda a figura. Neste caso foram consideradas janelas de 1 a 117 pixels.

será dada uma maior atenção a Santa Cruz do Sul, devido à sua situação de principal polo regional, concentrando as principais indústrias de fumo, principal produto agrícola da região. A partir de uma primeira análise de Santa Cruz do Sul será possível estabelecer um conjunto de parâmetros que sirvam de referência para a análise dos resultados das demais cidades que fazem parte do seu sistema regional. Santa Cruz do Sul é um dos principais núcleos da colonização alemã do Rio Grande do Sul. A colónia foi fundada, por lei provincial, a 6 de Dezembro de 1847. Esta cidade possui dois importantes limites: a leste, o morro chamado 'Cinturão Verde', e a oeste, a BR 471. Santa Cruz do Sul desenvolveu-se a partir de um núcleo planeado, com lotes de traçado regular (demarcados pelo engenheiro Frederico Cabral) e com um limite quadrangular - figuras 7 e 8 .

Até aos anos 20 do século passado, Santa Cruz manteve uma forma bastante regular. Ao longo do século $\mathrm{XX}$ a cidade foi-se expandindo seguindo antigas estradas e incluindo parcelas ligadas ao perímetro urbano anterior (Figura 7). Os morros foram sempre os 'constrangimentos' mais significativos, acabando por definir uma cidade com uma forma mais 'dendrítica'. Nos últimos anos, a cidade cresceu de forma rápida e pouco regulada. Esta particularidade é detetada através da observação dos valores de dimensão fractal que, em apenas 10 anos, aumentaram cerca de 25 por cento (Figura 8).

A criação do distrito industrial e a ocupação da Área de Preservação
Permanente - o Cinturão Verde contribuíram para este tipo de ocupação e para o crescimento do perímetro urbano. Outra característica fundamental de Santa Cruz do Sul é a ocupação de áreas próximas às suas principais saídas em direção às demais cidades do Vale do Rio Pardo, com uma forte ligação de complementaridade socioeconómica, provocando uma grande interação espacial. Esta interação ocorre diariamente, sendo que o fluxo de pessoas gerado se deve fundamentalmente a deslocações casa-trabalho, casa-estudo, local de produção-abastecimento, e por fim, ao acesso à oferta diversificada do seu polo principal, Santa Cruz do Sul. Naturalmente, esta rede afeta também as outras cidades, de dimensão mais reduzida, do Vale do Rio Pardo (Figura 9).

Muitos sistemas numa fase de crescimento apresentam auto-similaridade. Também a cidade tem sido considerada como um elemento com estas características. A similaridade pode também influenciar um conjunto de elementos que possuam ligação entre si, assim como as interações espaciais encontradas em sistemas regionais. Nas últimas décadas do século XX, uma parte da comunidade científica, na área da Física, começou a interessar-se pela dinâmica de sistemas ditos complexos, cujas partes interagem de forma não-linear. Uma das propriedades marcantes de tais sistemas é a presença de leis de escala ou Leis de Potência. Uma Lei de Potência é uma relação funcional entre duas quantidades. Por exemplo: a relação de complementaridade 


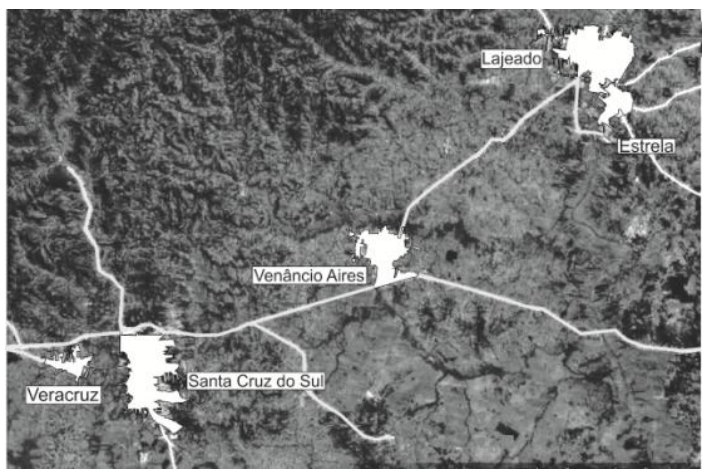

Figura 6. Distribuição territorial das cidades estudadas.

socioeconômica existente num sistema regional, regido por um polo principal, que pode afetar as demais cidades da rede, num determinado atributo. Neste caso, o atributo é a forma urbana e a ocupação urbana, medida pela dimensão fractal de cada cidade analisada.

A hipótese principal é que se um determinado tipo de ocupação começa a desenvolver-se numa cidade que é considerada um polo de um sistema regional - como é o caso de Santa Cruz do Sul outras cidades da mesma região poderão vir a apresentar o mesmo tipo de crescimento e ocupação do solo, gerando assim características formais similares. A dimensão fractal de cada uma das cidades permite comparar o grau de fragmentação do tecido urbano gerado pela ocupação recente - que define sua forma atual - tendo em consideração o seu perímetro urbano e os vazios internos.

Apesar de Santa Cruz do Sul ter mantido, ao longo de muitas décadas, uma forma compacta e regular semelhante ao seu núcleo inicial, com as mudanças na região e com o aparecimento de novos loteamentos e novos tipos habitacionais (por exemplo, os condomínios fechados), a cidade passou a apresentar uma forma dendrítica.

No contexto regional do Vale do Rio Pardo, o fenómeno de ocupação do solo urbano pode ser considerado como 'coevolução', ou seja, um processo evolucionário que surge da interação entre componentes de espécies diferentes num mesmo sistema - neste caso, as cidades da região que têm apresentado mudanças na sua forma urbana resultantes de novas ocupações do solo e de uma expansão incontrolada da área urbana. Nas cidades estudadas é possível observar um parcelamento de propriedades próximas às áreas já urbanizadas, e o desenvolvimento urbano ao longo de eixos fundamentais de transporte.

A uma escala menor, o crescimento envolve transições individuais relacionadas com o uso do solo, a ocupação e a densidade urbana. Se há uma alteração na configuração deste conjunto de componentes, o sistema urbano resultante deixa de apresentar apenas as propriedades coletivas dos componentes, passando também a incluir propriedades emergentes do sistema decorrentes de alterações das relações entre os componentes da estrutura urbana. Neste caso, a Lei de Potência, gerada por novas formas de ocupação urbana, leva a que no contexto regional se encontrem padrões similares. Isto mesmo é visível nas Tabela 1 e 2, que apresentam os resultados da análise da dimensão fractal das cinco cidades analisadas.

No caso da cidade de Lajeado, a ocupação urbana possuiu outro grau de organização, com uma alta dimensão fractal, próxima de 2. Como tal, poderia ser considerada uma figura totalmente ocupada. A contradição, porém, é a formação de um tecido urbano com pouca acessibilidade, com muitos loteamentos, com um modelo do tipo 'espinha de peixe' que está associado a uma elevada segregação.

As Leis de Potência revelam regularidades em propriedades de sistemas complexos - como são as cidades - que sofrem transformações em diferentes escalas e que apresentam similaridades entre elas. A visão numa determinada escala pode ser similar à visão noutras escalas. Os exemplos apresentados nas primeiras secções do artigo apresentam uma auto-similaridade perfeita por se tratarem de modelos matemáticos. No entanto, isto não acontece nem no ambiente urbano nem na natureza.

Conforme foi dito anteriormente, em muitos casos, as áreas urbanas são formadas por clusters de diferentes tamanhos, distribuídos espacialmente de um modo não homogéneo ao longo de vales, linhas de transporte e áreas menos acessíveis, que são ocupadas de maneira esparsa. Uma mudança de escala, da urbana para a regional, e considerando as diferentes cidades 


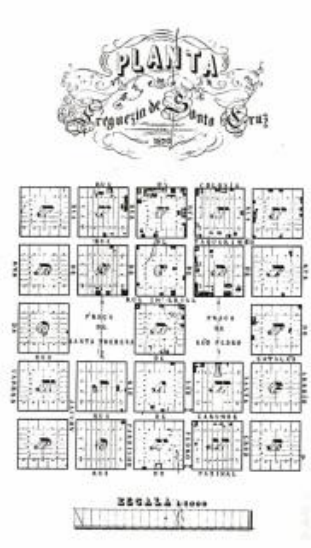

1870

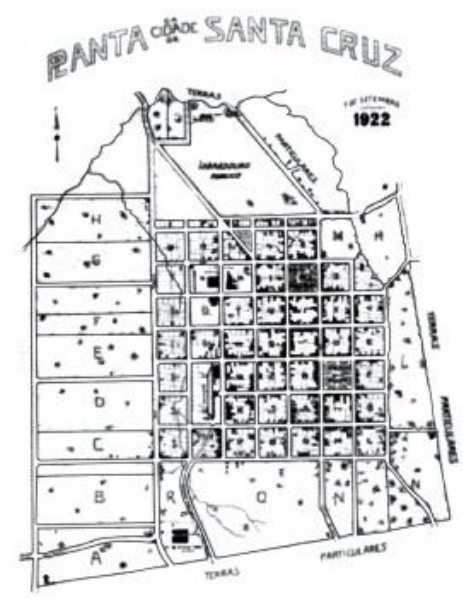

1922

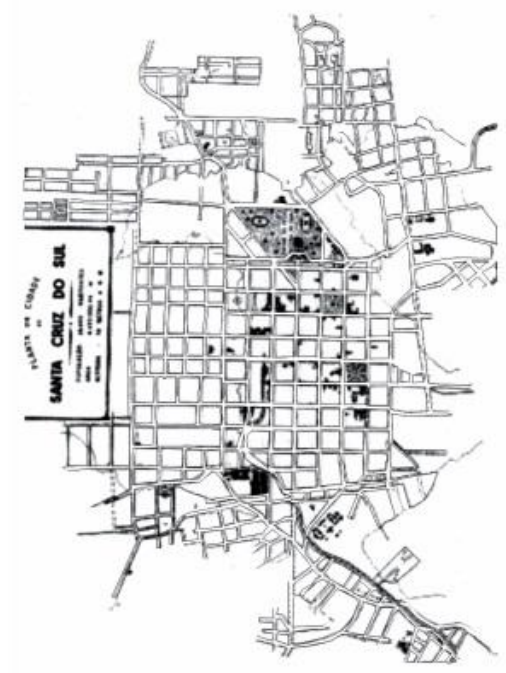

1957

Figura 7. Plantas de Santa Cruz do Sul em 1870, 1922 e 1957.

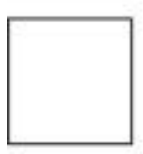

1870

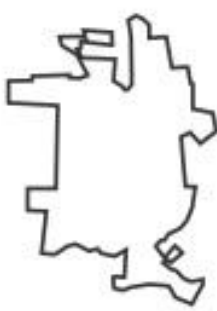

1957

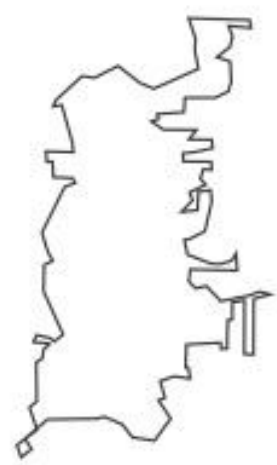

2000

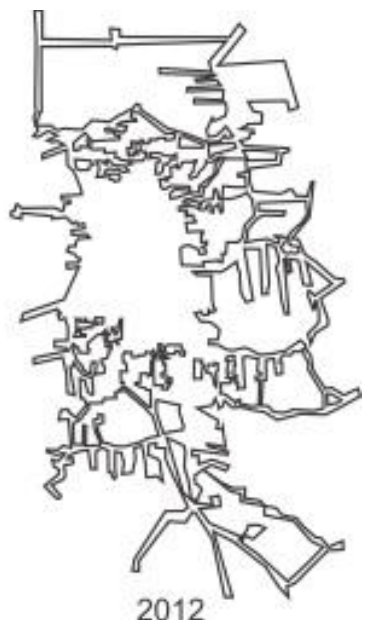

2012

Figura 8. Perímetro urbano de Santa Cruz do Sul entre 1870 e 2012.

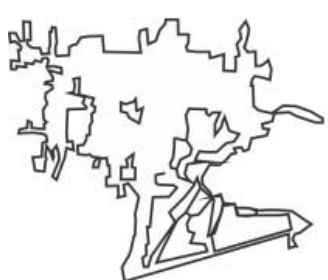

Venâncio Aires

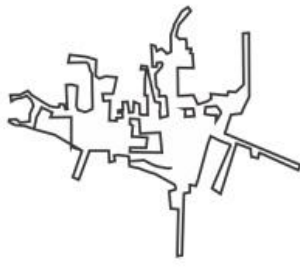

Veracruz

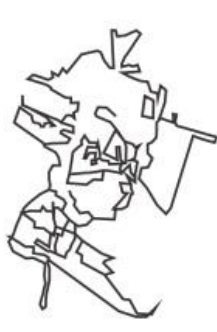

Estrela

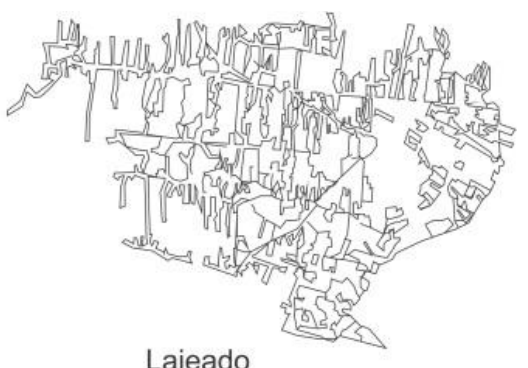

Lajeado

Figura 9. Contorno e vazios internos das cidades de Venâncio Aires, Vera Cruz, Estrela e Lajeado. 


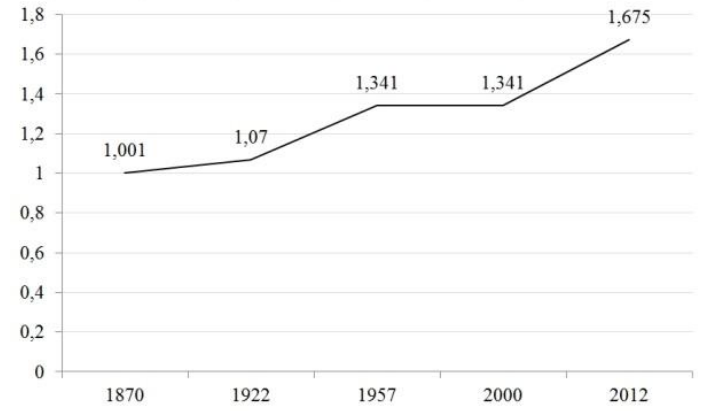

Tabela 1. Dimensão Fractal de Santa Cruz do Sul entre 1870 e 2012.
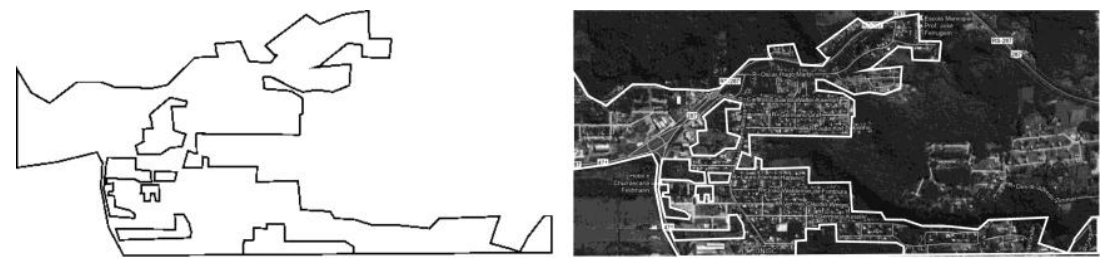

Figura 10. Parcela da cidade de Santa Cruz do Sul.
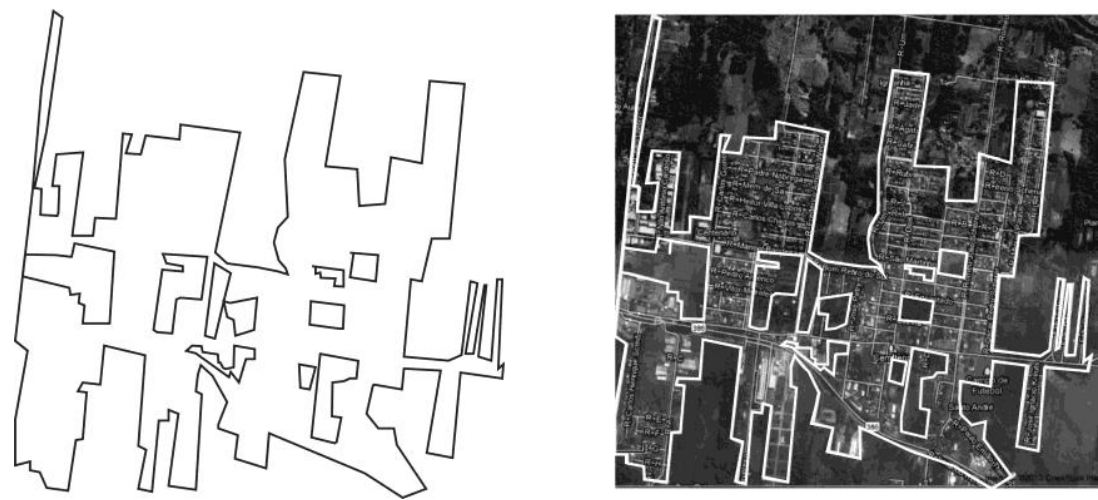

Figura 11. Parcela da cidade de Lajeado.

integrantes do Vale do Rio Pardo, evidencia todo um conjunto de semelhanças no tipo de crescimento urbano. Por outro lado, a característica básica de um fractal é a autosimilaridade em diferentes escalas que pode passar do âmbito regional para o urbano e para o intraurbano.

\section{Escala intraurbana}

A análise dos limites de uma cidade revela

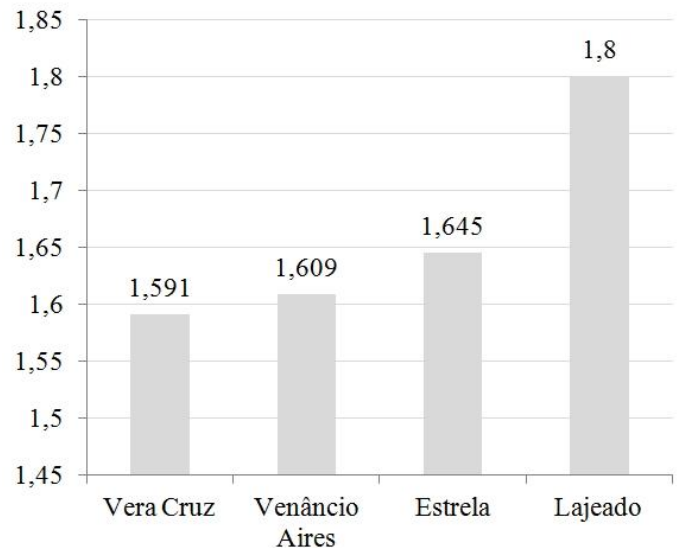

Tabela 2. Dimensão fractal de Venâncio Aires, Vera Cruz, Estrela e Lajeado. 
ruas e quarteirões, a alta fragmentação do tecido urbano permaneceria (mantendo-se também no perímetro urbano como um todo).

No caso de Santa Cruz do Sul existe autosemelhança quando se parte para escalas menores de observação intraurbana. A dimensão fractal da parcela estudada nesta cidade é 1,565, quando analisado o perímetro urbano e as áreas de ocupação mais rarefeita (Figura 10). Do mesmo modo, também no caso da parcela da cidade de Lajeado a dimensão fractal continua elevada, com 1,567 (Figura 11).

Os exemplos das parcelas de Santa Cruz do Sul e Lajeado mostram que, mesmo alterando a escala de observação do território, é possível observar que os impactos gerados no tecido urbano são semelhantes às análises anteriores desenvolvidas a escalas maiores - desde o desenvolvimento de uma forma dendrítica, até à incompatibilidade dos novos loteamentos com a rede viária existente.

\section{Conclusões}

Com o estudo de caso aqui apresentado, foi possível demonstrar a validade de uma metodologia de análise dos fenómenos urbanos, centrada na forma urbana e com uma forte componente de geometria fractal. Neste caso, o grau de fragmentação e preenchimento urbano pode ser avaliado a partir da dimensão fractal que, em Santa Cruz do Sul, contribui para a compreensão da dinâmica espacial e temporal da sua forma urbana. Com essa abordagem foi também possível analisar a dinâmica regional de expansão urbana de um conjunto de cidades inter-relacionadas por complementaridades socioeconómicas no Vale do Rio Pardo.

Com base na dimensão fractal identificaram-se padrões de crescimento semelhantes associados à expansão desregrada, vigente na região, que leva à fragmentação dos tecidos urbanos e à emergência de formas urbanas irregulares. Essa propriedade foi detectada através da aplicação da dimensão fractal, mostrando-se tanto em âmbito intraurbano (indicando a existência de descontinuidades e vazios) como em perímetros irregulares (que se repetem, em maior ou menor grau, nas cidades analisadas).

\section{Referências}

Allen, P. M. (1997) Cities and regions as selforganizing systems: model of complexity (Gordon and Breach Science Publishers, Amsterdão).

Azevedo, T. e Christofoletto, A. (2007) 'Fractais em geografia, conceitos e perspectivas' Climatologia e Estudos da Paisagem 2, 30-49.

Batty, M. (2003) 'Agent-based pedestrian modelling' in Longley, P. e Batty, M. (eds.) Advanced spatial analysis, the CASA book of GIS (ESRI Press, Redlands).

Batty, M. e Longley, P. A. (1994) Fractal cities: a geometry of form and function (Academic Press, Londres).

Benguigui, L., Blumenfeld-Lieberthal, E. e Batty, M. (2008) 'Macro and micro dynamics of city size distributions: The case of Israel', CASA Working Paper 139.

Cooper, J. e Oskrochi, R. (2008) 'Fractal analysis of street vistas: a potential tool for assessing levels of visual variety in everyday street scenes', Environment and Planning B: Planning and Design 35, 349-63.

Falconer, K. J. (1990) Fractal geometry: mathematical foundations and applications (John Wiley \& Sons, Londres).

Feng, J. e Chen, Y. (2010) 'Spatiotemporal evolution of urban form and land-use structure in Hangzhou, China: evidence from fractals', Environment and Planning B: Planning and Design 37, 838-56.

Frankhauser, P. (1994) La fractalité des structures urbaines (Anthropos, Paris).

Frankhauser, P. (1998) 'The fractal approach. A new tool for the spatial analysis of urban agglomerations. Special Issue on Population', New Methodological Approaches in the Social Sciences 10, 205-40.

Frankhauser, P. e Pumain, D. (2002) 'Fractales et géographie', in Sanders, L. (ed.) Modèles en analyse spatiale (Lavoisier, Paris) 301-29.

Frankhauser, P. (2004) 'Comparing the morphology of urban patterns in Europe a fractal approach' in Borsdorf, A. e Zembri, P. (eds.) Report COST Action 10 Urban Civil Engineering (COST, Bruxelas) 79-105.

Joye, Y. (2011) 'A review of the presence and use of fractal geometry in architectural design', Environment and Planning B: Planning and Design 38, 814-28.

Mandelbrot, B. (1982) The fractal geometry of nature (W H Freeman, Nova Iorque).

Terzi, F. e Kaya, H. S. (2011) 'Dynamic spatial 
analysis of urban sprawl through fractal geometry: the case of Istanbul', Environment and Planning B: Planning and Design 38, 17590.

Trentin, G. (2012) Dimensão fractal, dinâmica especial e padrões de fragmentação urbana de cidades médias do estado de São Paulo (Instituto de Geociências, São Paulo).

Weidlich, W. (2000) Sociodynamics: a systematic approach to mathematical modelling in the social sciences (Wolfgang Harwood Academic Publishers, Amsterdão).

\title{
Tradução do resumo e palavras-chave
}

\begin{abstract}
Conventional methodologies in the study of urban form have not been considering the whole morphological complexity of cities or the degree of irregularity of urban perimeters as they address the physical form of cities within the scope of Euclidean geometry. This paper explores the application of fractal geometry in the study of urban areas, focusing on a number of aspects of physical form related to the fragmentation of urban fabrics, the presence of urban voids and, finally, the existence of selfsimilarity at different scales of observation. This analysis draws on the research work developed by Frankhauser from the 1990s onwards. Fractal analysis is applied to a set of five cities in the Vale do Rio Pardo, Rio Grande do Sul, Brazil. Over the last years, this set of cities had an uncontrolled pattern of growth. The rapid urban development patterns and the expansion of urban perimeters have led to a type of rarefied territorial occupation with major impacts on the urban fabrics of these cities.
\end{abstract}

Key Words: Urban studies, fractal geometry, fractal dimension, self-organization, Vale do Rio Pardo

\section{Turkish Network of Urban Morphology}

A primeira reunião da Turkish Network of Urban Morphology (TNUM) teve lugar no dia 11 de Abril de 2014 no Center for Mediterranean Urban Studies da Mersin University, em Mersin. Os objetivos fundamentais desta reunião foram os seguintes: formar um espaço comum para a partilha de diferentes estudos morfológicos; discutir as possibilidades de desenvolvimento de estudos colaborativos, na área da forma urbana, por diferentes investigadores e instituições turcas; e, por fim, alargar os estudos a um nível internacional através da cooperação com outras redes nacionais e regionais e com o International Seminar on Urban Form (ISUF).

A primeira reunião da rede, designada como Foundation Workshop, foi palco de muitas e proveitosas discussões. Na primeira parte do workshop os vários investigadores tiveram oportunidade de partilhar os seus estudos e análises. A segunda parte foi dedicada a um debate sobre o futuro do TNUM e sobre os diferentes desenvolvimentos possíveis para esta nova rede de investigação.

Sublinham-se três aspetos fundamentais deste debate. $\mathrm{O}$ primeiro refere-se à estrutura organizacional da rede. Foi definido que o Center for Mediterranean Urban Studies irá atuar como elemento coordenador do TNUM. Os investigadores que estiveram presentes no workshop farão parte de uma comissão diretiva presidida por Ayşe Sema Kubat (Department of Urban and Regional Planning, Istanbul Technical University) coadjuvada pelo diretor do Center for Mediterranean Urban Studies, Tolga Ünlü (Department of City and Regional Planning, Mersin University).

$\mathrm{O}$ segundo aspeto que importa referir prende-se com a formação de uma comissão com o objetivo de preparar uma lista bibliográfica de estudos sobre forma urbana na Turquia. Este trabalho terá por base o artigo de Ayşe Sema Kubat, The study of urban form in Turkey, publicado na revista Urban Morphology (Kubat, 2010).

Por fim, foi estabelecido que o primeiro simpósio do TNUM realizar-se-á na Mersin University, a 22 e 23 de Outubro de 2015, e irá discutir as diferentes abordagens e perspetivas no estudo da forma urbana na Turquia.

Reference

Kubat, A. S. (2010) 'The study of urban form in Turkey', Urban Morphology 14, 31-48.

Tolga Ünlü, Department of City and Regional Planning, Mersin University, Yenisehir, Mersin 33343, Turkey. Email: tolgaunlu@gmail.com 